\title{
Call Center Remote Triage by Nurse Practitioners Was Associated With Fewer Subsequent Face-to-Face Healthcare Visits
}

\author{
Peter J. Kaboli, MD, MS ${ }^{1,2}$, Matthew R. Augustine, MD, $M S^{3,4}{ }_{(0)}$, \\ Danielle E. Rose, $P h D, M P H^{5}$, Neetu Chawla, $P h D^{5}$, Maria Bouchard, MN, FNP-BC $C^{6}$, and \\ Paul Hebert, $P h D^{7}$
}

\begin{abstract}
'Veterans Rural Health Resource Center-lowa City, VA Office of Rural Health, and Center for Access and Delivery Research and Evaluation (CADRE) at the lowa City VA Healthcare System, lowa City, IA, USA; ${ }^{2}$ Department of Internal Medicine, University of lowa Carver College of Medicine, lowa City, IA, USA; ${ }^{3}$ Department of Medicine, Geriatric Research Education and Clinical Center, James J Peters VA Medical Center, Bronx, NY, USA; ${ }^{4}$ Department of Medicine, Icahn School of Medicine at Mount Sinai, New York, NY, USA; ${ }^{5}$ Center for the Study of Healthcare Innovation, Implementation and Policy, VA HSR\&D, VA Greater Los Angeles Healthcare System, Los Angeles, CA, USA; ${ }^{6}$ VISN 22 Clinical Contact Center, Greater Los Angeles-Sepulveda Advice Call Center, North Hills, CA, USA; ${ }^{7}$ VA Puget Sound Health Care System, Seattle, WA, USA.
\end{abstract}

BACKGROUND: In 2015, the Veterans Health Administration (VHA) incorporated nurse practitioners (NPs) into remote triage call centers to supplement registered nurse (RN)-handled calls.

OBJECTIVE: To assess 7-day healthcare use following telephone triage by NPs compared to RNs. We hypothesized that NP clinical decision ability may reduce followup healthcare.

DESIGN: Retrospective observational comparative effectiveness study of clinical and administrative databases. NP routed calls were matched to RN calls based on chief complaint with propensity score matching and multivariate count data models, adjusting for differences in call severity and patient comorbidity.

PARTICIPANTS: Callers to a VHA regional call center, April 2015 to March 2019.

MAIN MEASURES: Primary care, specialty care, and emergency department (ED) visits plus hospitalizations within 7 days.

KEY RESULTS: NP-handled calls $(N=1554)$ were matched to RN calls $(N=48,024)$ for the same chief complaint. NP-handled calls, compared to RNs, had lower comorbidities, fewer hospitalizations, and less urgent complaints. Seven-day healthcare use was lower for NP compared to RN calls for specialty care ( $0.15 \mathrm{vs.} 0.20$ visits per person [VPP]; $p<0.001$ ), ED (0.11 vs. $0.27 \mathrm{VPP} ; p<$ 0.001 ), and hospitalizations (0.01 vs. 0.04 VPP; $p<$ $0.001)$, but not primary care (0.43 vs. 0.42 VPP; $p=$ 0.80 ). In adjusted analyses, estimated avoided in-person visits per 100 calls routed to NPs were 0.7 primary care visits $(95 \%$ confidence interval $[\mathrm{CI}] 0.4,1.0), 2.6$ specialty care visits (95\% CI 0.0, 5.1), 5.9 ED visits (95\% CI 2.7, 9.1), and 1.4 hospital stays (95\% CI 0.1, 2.6). Propensity score-matched models comparing NP $(N=1533)$ to RN $(N$ = 2646) calls had adjusted odds ratios for 7 -day

This manuscript is not under review elsewhere and there is no prior publication of manuscript contents.

Received July 8, 2020

Accepted December 21, 2020

Published online January 26, 2021 healthcare use of 0.75 (primary care), 0.75 (specialty care), and 0.73 (ED) (all $p<0.003$ ).

CONCLUSION: Incorporating NPs into a call center was associated with lower in-person healthcare use in the subsequent 7 days compared to routine $\mathrm{RN}$-triaged calls.

KEY WORDS: after-hours care; telephone triage; call center; telephone medicine.

$\mathrm{J}$ Gen Intern Med 36(8):2315-22

DOI: $10.1007 / \mathrm{s} 11606-020-06536-0$

() This is a U.S. government work and not under copyright protection in the U.S.; foreign copyright protection may apply 2021

\section{BACKGROUND/INTRODUCTION}

Call center remote triage services in healthcare refer to the initial assessment and management of acute, undifferentiated, or unscheduled clinical care initiated by a patient or family member from a distance. ${ }^{1}$ Historically, registered nurses (RNs) with clinical expertise in phone triage and/or evaluation books or software to guide recommendations have performed most remote triage. Potential benefits of remote triage include reduced medical workload, improved access to primary care (PC) advice, reduced travel burden, and improved resource utilization of urgent and emergency department (ED) services. However, based upon a recent systematic review, limited evidence supports reductions in subsequent care, and some studies actually found increased healthcare use. ${ }^{1,2}$

The primary promise of remote triage for healthcare systems is to appropriately refer patients to routine in-person PC visits for those requiring it, while also reducing costly inappropriate urgent or ED care. When evaluating downstream PC use, only one study from the UK's National Health Service (NHS) demonstrated reduced visits after the initiation of a telephone triage system. ${ }^{3}$ In four randomized controlled trials, two resulted in increases in PC visits, ${ }^{4,5}$ while another two showed no downstream effect. ${ }^{6,7}$ For ED visits, three studies 
of nurse triage vs. usual care showed no reduction in ED visits. $^{4,5,8}$

The clinical training of the phone call handler may be an important component of remote triage and mitigating unneeded health service utilization. One 3-arm cluster randomized trial in the UK compared RNs to general practitioners (GPs) to usual care and found the GP-led triage resulted in fewer mean number of $\mathrm{PC}$ visits in the 28 days after the phone call compared to RNs. ${ }^{4}$ This difference was potentially due to the ability of the physician to prescribe treatment for the condition over the phone and avoid an inperson visit. A second study evaluated a new NHS phone triage system that employed non-clinical call handlers to use computerized decision support to triage calls directly to the most appropriate service, including on-site clinicians who could address the immediate care need. ${ }^{9}$ This new NHS "111" service resulted in an average of 47 extra PC visits per 1000 triaged calls (95\% CI -66 to 156 ).

The Veterans Health Administration (VHA) is the largest integrated healthcare system in the USA with over 9 million enrolled Veterans. Due to shared electronic health records (EHR), ability to practice across state lines, and limited third-party billing, VHA has a vested interest in providing the appropriate level of care at the most convenient time for patients to achieve the best possible outcome. Thus, in 2015 , VHA initiated a pilot program to incorporate nurse practitioners (NPs) into a Veterans Integrated Service Network (VISN) 22 regional clinical call center at the Greater Los Angeles (GLA) VA Medical Center with the goal of increasing clinical capacity to handle clinical concerns remotely and avert unnecessary healthcare use. The overall objective of this study was to determine the downstream clinical use of in-person care after the initiation of an NP-led triage system. Our hypothesis was NP-handled calls would result in reduced healthcare use within 7 days compared to calls handled by nurses. A secondary objective was to describe the types of calls handled by NPs through this enhanced call center system.

\section{METHODS}

This was a retrospective observational comparative effectiveness study of VHA administrative and clinical databases.

\section{Subjects and Setting}

All callers to the VISN 22 Nurse Advice Call Center (VNACC) from facilities where NPs were credentialled from April 2015 to March 2019 were eligible for inclusion in the study sample. The VNACC has provided registered nurse $(\mathrm{RN})$ triage for over two decades in a hub-and-spoke model with GLA as the hub providing service to up to 26 hospitals and clinics. NP care was initiated at GLA and expanded to spoke sites of the San Diego, Long Beach, and Loma Linda, CA and Las Vegas, NV healthcare systems, serving 1.5 million Veterans. The incorporation of NP care and changes in $\mathrm{RN}$ processes required ongoing education and trainings, relationship building, and process improvement over time.

To guide the call center operator, the program used an established call triage protocol (Fig. 1). Emergent calls, defined by a set of criteria aligned with an Emergency Severity Index (ESI) $\operatorname{score}^{10}$ of $1-3$, were routed to an RN directly through a warm handoff. Non-emergent calls, defined by an ESI score of 4 or 5, were placed in a queue for callback within 15-120 min. All non-emergent calls were routed to an RN equipped with Triage Expert Dual Purpose (TEDP) software to guide disposition. Subsequent referral to the NP was nonrandom since this could only happen during hours an NP was available or was not busy with another patient. For patients referred to the NP, care included medical treatment with diagnostics and prescriptions as clinically indicated and potential downgrading of patient disposition from the conservative triage algorithms (e.g., from next day in-person follow-up to telephone only follow-up). Additionally, if an in-person visit was indicated, the NP could pre-order diagnostic tests to facilitate one-point decision-making for the patient and inperson clinician.

Initial NP hours were 8:00 am-4:00 pm Monday through Friday excluding holidays. Extended NP hours with staggered



Figure 1 Flow diagram of patient experience when contacting call center. 
shifts to cover 6:00 am-7:00 pm coincided with spoke site expansion. VNACC NP staffing from 2015 to mid-2017 was a total 1.75 full-time equivalent employees (FTEE) with other responsibilities including staffing an in-person clinic and administrative duties; a total of 1.5 FTEE NP was present from 2017 to 2019 .

\section{Data Sources}

The primary source of data was generated by the Triage Expert Dual Purpose (TEDP) software. ${ }^{11}$ TEDP is a clinical software application that allows RNs to systematically assess a caller's symptoms and make recommendations for subsequent care. RNs select from a list of 850 chief complaints the one that best matches the caller's symptoms, and a computer algorithm guides the $\mathrm{RN}$ to solicit other information relevant to that condition. These include the duration of the complaint, presence of other illnesses relevant to the chief complaint, and physiological measures such as pain on a $0-10$ scale. The algorithm then makes recommendations for follow-up care timing that ranges from calling a 911 emergency to self-care, and a recommended follow-up location, from ED to home. The software places a text file with this information in the EHR, which is stored in the VHA Corporate Data Warehouse (CDW). Data on patient characteristics and VHA healthcare use subsequent to the call also come from the CDW.

\section{Outcome Measures}

Outcomes included healthcare use within 7 days of the call to the remote triage call center. We identified all in-person primary care, specialty care, and ED visits as well as hospital admissions occurring at VHA facilities and non-VHA locations paid by VHA through fee-basis care.

\section{Primary Exposure}

The primary exposure was an indicator for whether the call was routed to an NP or handled routinely by an RN.

Patient-Level Variables. Patient demographics include age, race, sex, Gagne comorbidity score ${ }^{12}$ (derived from diagnosis codes generated from inpatient and outpatient visits in the year prior to the call), percent service-connected disability, urban/ rural residence, marital status, and prior primary and specialty outpatient, ED, and hospital use.

Call-Level Variables. From the TEDP note, we abstracted the chief complaint including duration, number of other complaint-relevant conditions, and the pain score. We used the recommended follow-up interval and the recommended follow-up location to classify calls into four categories of disposition urgency: ED, urgent care, clinic today, or after today/by phone. Although the call center is available $24 \mathrm{~h}$ per day, 7 days per week, most calls occurred during business hours from 8:00 am to 4:00 pm. Typically, calls on Monday and calls earlier in the day tend to be for more urgent symptoms so day and time of the call were included and used in matching.

\section{Statistical Analyses}

To limit the number of $\mathrm{RN}$ calls that could potentially be compared, we restricted the database to calls that could have been handled to an NP. We listed chief complaints for calls handled by NPs, then restricting our sample to calls for those chief complaints, regardless of whether they were handled by an RN or NP. We also restricted the days of the week and time of day to hours when NPs worked.

We performed 2 sets of statistical analyses. First, multivariate logit models were used to estimate the association between NP vs. routine RN-handled calls and the probability of any visit to the ED, primary care, specialty care, or hospitalization, respectively, within 7 days of the index call. Control variables were patient-level and call-level variables described above. We also estimated Poisson models where the dependent variable was the total number of visits over 7 days for each caller for the same types of visits and independent variables as the logit models. Because the distribution of visits to specialty care was over-dispersed, we estimated a negative binomial model for specialty care visits. We report the adjusted odds ratios from the logit models and adjusted rate ratios from the Poisson and negative binomial models. We also use the parameters from the count data models to estimate the number of visits avoided per 100 calls routed to an NP. We estimated the standard error for avoided visits using the delta method. $^{13}$

Second, we used propensity score matching to match NP calls to routine RN-handled calls. We estimated an antiparsimonious logistic regression where NP vs. RN call was the dependent variable and patient and call characteristics were independent variables. These included comorbidity score, demographics, marital status, rurality, priority status (i.e., \% service connected disability), prior healthcare use, day of call, time of day of the call, the pain score, distance from the callers residence to the VA, and the number of other present conditions related to the primary complaint of the call. To reduce the imbalance in urgency between NP and routine RN calls, we also included interactions between urgency and patient age, marital status, prior healthcare use, comorbidity, pain, and the number of other present conditions. We used 2:1 nearest neighbor matching without replacement to match each NP call to 2 routine calls that were for the same chief complaint as the NP call and were within a caliper of $1 / 5$ th of a standard deviation of the propensity score. ${ }^{14}$ We also limited the sample to calls that were in the common support of the distributions of propensity scores for NP and routine RN calls within each chief complaint. Because balancing tests suggested imbalance in urgency after matching, we estimated the logistic and count data models described above with an 
indicator for NP vs routine $\mathrm{RN}$ call and indicators for the urgency of the call as independent variables. The resulting, smaller, sample size had too few inpatient visits within 7 days of the index calls to estimate the association of NP-handled calls with hospitalizations.

The authors had full access to and take full responsibility for the integrity of the data. All analyses were conducted using Stata $15 .{ }^{15}$ The study was reviewed by the University of Iowa Institutional Review Board and the Iowa City VA Healthcare System Research and Development Committee.

\section{RESULTS}

Over the study period, NPs triaged 1554 calls and RNs triaged 48,024 calls with the same chief complaints for a total of 49,578 calls included in this study. Overall, patients calling had a mean age of 57.2 (SD 17.2) years; $14.0 \%$ were female; $56 \%$ were white; $46 \%$ were married; and $86.5 \%$ lived in an urban area. Patients routed to NPs for triage were slightly older (57.3, SD 17.2 vs. 54.4, SD 17.5 years) and more likely to be male, white, married, and living in rural areas (Table 1). Furthermore, patients triaged by NPs had lower comorbidity and lower prior healthcare use, including ED visits and hospitalizations in the prior 12 months. The number of outpatient visits in the prior year was not statistically different. NPs were more likely to handle calls with TEDP triage disposition of lower urgency. Calls handled by NPs were less likely to have disposition to ED (2.19\% vs. $30.1 \%$ ) and more likely to have a disposition to clinic or phone visit after the day of the call (64.2\% vs. $32.6 \%)$. Differences in chief complaint category were notable; NP calls more likely to be for respiratory and urinary symptoms.

Figure 2 shows healthcare use, adjusted for differences in symptom severity and the callers' comorbidity and prior healthcare use, in the 7 days following calls for chief complaints that were handled by both NPs and RNs and that occurred during hours when NPs were working. Only ED visits on the day of the call were statistically significantly lower for NP-triaged calls. However, the probability of any use and the count of visits over 7 days were statistically significantly lower for NP-triaged calls for primary care, specialty care, and ED visits, but not for hospitalizations (Table 2). For example, $23 \%$ of callers managed by RNs had an ED visit in the 7 days following the call, compared with $9 \%$ of callers managed by an NP for an adjusted OR of $0.71(p<0001)$. The

Table 1 Descriptive Statistics of Calls Handled by Registered Nurses (RNs) and Nurse Practitioners (NPs) with Same Chief Complaints from 2015 to 2019

\begin{tabular}{|c|c|c|c|c|}
\hline & $\begin{array}{l}\text { Total } \\
(N=49,578)\end{array}$ & $\begin{array}{l}\text { NP calls } \\
(N=1554)\end{array}$ & $\begin{array}{l}\text { RN calls } \\
(N=48,024)\end{array}$ & $p$ value \\
\hline Mean age (SD) & $57.2(17.2)$ & $54.4(17.5)$ & $57.3(17.2)$ & $<0.001$ \\
\hline Female, \% & 14.0 & 18.5 & 13.8 & $<0.001$ \\
\hline \multicolumn{4}{|l|}{ Race, $\%$} & 0.084 \\
\hline White & 56.0 & 53.8 & 56.1 & \\
\hline Black & 15.0 & 15.2 & 15.0 & \\
\hline Hispanic & 14.7 & 14.3 & 14.7 & \\
\hline Other/missing & 14.3 & 16.7 & 14.2 & \\
\hline \multicolumn{4}{|l|}{ Marital status, \% } & $<0.001$ \\
\hline Married & 46.0 & 42.9 & 46.1 & \\
\hline Divorced/separated & 34.7 & 33.6 & 34.8 & \\
\hline Never married/missing & 19.3 & 23.5 & 19.1 & \\
\hline \multicolumn{4}{|l|}{ Rurality } & 0.003 \\
\hline Urban & 86.5 & 89.1 & 86.4 & \\
\hline Rural & 9.96 & 7.01 & 10.07 & \\
\hline Missing & 3.47 & 3.80 & 3.46 & \\
\hline Service connected, \% & 42.6 & 43.3 & 42.5 & 0.43 \\
\hline Comorbidity score, mean (SD) & $0.46(1.34)$ & $0.27(1.00)$ & $0.46(1.35)$ & $<0.001$ \\
\hline \multicolumn{5}{|l|}{ Healthcare use, prior 12 months } \\
\hline Outpatient visits, mean (SD) & $9.2(12.8)$ & $8.6(11.7)$ & $9.2(12.8)$ & 0.10 \\
\hline ED visits, mean (SD) & $0.73(1.7)$ & $0.50(1.2)$ & $0.74(1.7)$ & $<0.001$ \\
\hline Hospitalization, mean (SD) & $0.17(0.62)$ & $0.10(0.45)$ & $0.17(0.63)$ & $<0.001$ \\
\hline \multicolumn{4}{|l|}{ TEDP triage disposition, $\%$} & $<0.001$ \\
\hline After today or phone & 33.6 & 64.2 & 32.6 & \\
\hline Clinic today & 30.9 & 24.4 & 31.1 & \\
\hline Urgent care & 3.80 & 3.70 & 3.78 & \\
\hline Emergency department & 29.3 & 2.19 & 30.1 & \\
\hline Missing & 11.2 & 23.5 & 10.8 & \\
\hline \multicolumn{4}{|l|}{ Chief complaint category, $\%$} & $<0.001$ \\
\hline Pain & 40.8 & 33.5 & 41.0 & \\
\hline Respiratory & 15.7 & 33.3 & 15.1 & \\
\hline Cardiac & 8.06 & 2.45 & 8.25 & \\
\hline Urinary & 7.88 & 15.8 & 7.62 & \\
\hline Ear, nose, throat & 7.82 & 3.41 & 7.96 & \\
\hline Dermatologic & 7.09 & 1.54 & 7.27 & \\
\hline Other & 8.45 & 7.40 & 8.49 & \\
\hline
\end{tabular}

TEDP Triage Expert Dual Purpose software 


\section{Emergency Department}



Specialty Care

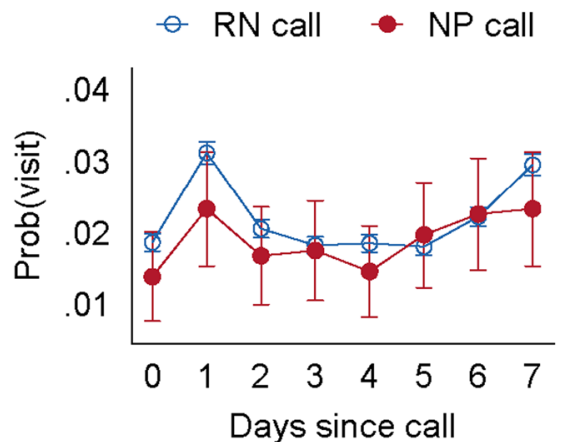

Primary Care

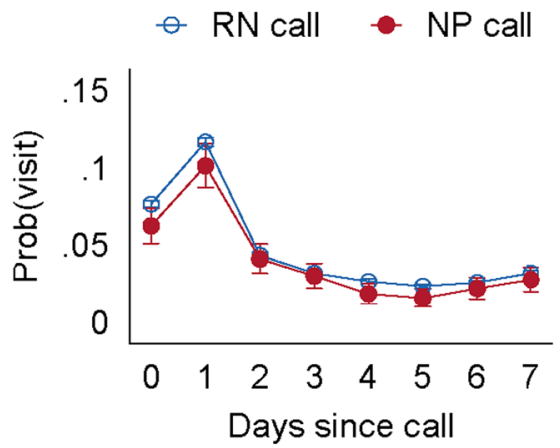

Hospitalization

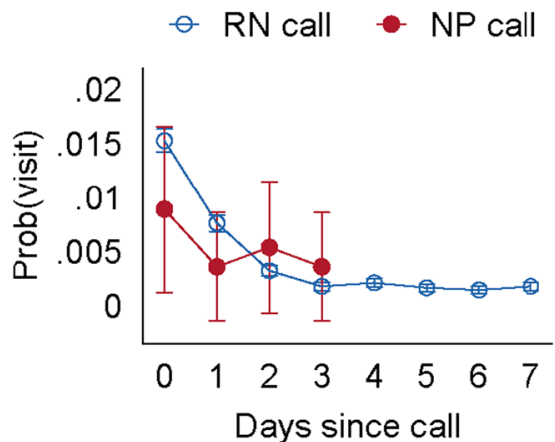

Fig. 2 Adjusted probability of healthcare use by day, type of use, and whether call was routed to an NP or RN. Calls to an NP were matched to RN routed calls based on the chief complaint of the call and whether the call was received during normal working hours. Estimates are adjusted for probability of visit from logistic regressions that controlled for patient socio-demographics, duration of the complaint, urgency of the condition as defined by the triage software, patient-reported pain score, and number of complicating factors the patients identified.

mean number of ED visits in the 7 days following the call was 0.27 for RN-triaged calls versus 0.11 for NP-triaged calls for an adjusted rate ratio of $0.78(p=0.002)$. Avoided in-person visits per 100 calls routed to NPs was estimated at 5.9 ED visits $(95 \%$ confidence interval $[\mathrm{CI}] 2.7,9.1), 2.6$ specialty care visits $(95 \%$ CI $0.0,5.1), 1.4$ hospital stays $(95 \%$ CI $0.1,2.6)$, and 0.7 primary care visits $(95 \%$ CI $0.4,1.0)$ (Table 2$)$.
Propensity score matching yielded similar findings (Table 3; Fig. 3). The propensity score--matched $\mathrm{RN}(N=2646)$ and NP $(N=1533)$ calls showed statistically significantly lower probability of any ED and specialty care visits over 7 days, respectively, for NP-triaged calls compared with RN-triaged calls. With adjustment for residual imbalance in urgency, the adjusted odds ratio became statistically significant for any primary care visit and remained statistically significant for any

Table 2 Probability of Healthcare Use at 7 Days Since Index Call (Unadjusted and Adjusted)

\begin{tabular}{|c|c|c|c|c|c|c|c|c|c|c|c|}
\hline & \multicolumn{5}{|c|}{ Probability of any use } & \multicolumn{6}{|c|}{ Count of use: visits per person (VPP) } \\
\hline & \multicolumn{3}{|c|}{ Unadjusted } & \multicolumn{2}{|c|}{ Adjusted* } & \multicolumn{3}{|c|}{ Unadjusted } & \multicolumn{3}{|c|}{ Adjusted* } \\
\hline & $\begin{array}{l}\mathrm{RN} \\
\text { call }\end{array}$ & $\begin{array}{l}\mathrm{NP} \\
\text { call }\end{array}$ & $p$ value & OR & $p$ value & $\begin{array}{l}\mathrm{RN} \\
\text { call }\end{array}$ & $\begin{array}{l}\text { NP } \\
\text { call }\end{array}$ & $p$ value & $\begin{array}{l}\text { Rate } \\
\text { ratio }^{\dagger}\end{array}$ & $p$ value & $\begin{array}{l}\text { Visits avoided } \\
\text { per } 100 \text { calls }(95 \% \mathrm{CI})\end{array}$ \\
\hline Primary care & 0.35 & 0.34 & 0.18 & 0.76 & $<0.001$ & 0.42 & 0.43 & 0.80 & 0.90 & 0.008 & $0.7(0.4,0.1)$ \\
\hline Specialty care & 0.15 & 0.11 & $<0.001$ & 0.78 & 0.002 & 0.20 & 0.15 & $<0.001$ & 0.87 & 0.04 & $2.6(0.0,5.1)$ \\
\hline $\mathrm{ED}$ & 0.23 & 0.09 & $<0.001$ & 0.71 & $<0.001$ & 0.27 & 0.11 & $<0.001$ & 0.78 & 0.002 & $5.9(2.7,9.1)$ \\
\hline Hospitalization & 0.03 & 0.01 & $<0.001$ & 0.64 & 0.13 & 0.04 & 0.01 & $<0.001$ & 0.60 & 0.08 & $1.4(0.1,2.6)$ \\
\hline
\end{tabular}

*Odds ratio from a logistic regression of any use as a function of patient characteristics (i.e., socio-demographics; prior 12-month emergency department, primary care, specialty care, and inpatient use), and call characteristics (i.e., chief complaint, duration of complaint, urgency of condition, and pain score)

${ }^{\prime}$ Rate ratio from a Poisson model of the same variables as the logistic regression. Because the distribution of specialty care visits showed evidence of overdispersion, rate ratios for specialty visits are from a negative binomial regression 
Table 3 Healthcare Use in the 7 Days Since Index Call: Propensity Score-Matched NP- and RN-Triaged Calls

\begin{tabular}{|c|c|c|c|}
\hline & $\begin{array}{l}\text { Primary } \\
\text { care }\end{array}$ & $\begin{array}{l}\text { Specialty } \\
\text { care }\end{array}$ & ED \\
\hline \multicolumn{4}{|c|}{ Probability of healthcare use at 7 days since index call } \\
\hline \multicolumn{4}{|c|}{ Propensity score matched } \\
\hline $\mathrm{RN}$ call $(N=2646)$ & 0.41 & 0.15 & 0.13 \\
\hline NP call $(N=1533)$ & 0.34 & 0.11 & 0.09 \\
\hline Odds ratio & 0.74 & 0.7 & 0.66 \\
\hline$p$ value & 0.15 & $<0.001$ & $<0.001$ \\
\hline \multicolumn{4}{|c|}{ Propensity score matched and adjusted } \\
\hline Adjusted odds ratio & 0.75 & 0.75 & 0.73 \\
\hline$p$ value & $<0.001$ & 0.003 & $<0.001$ \\
\hline \multicolumn{4}{|c|}{ Mean healthcare use per caller at 7 days since index call } \\
\hline \multicolumn{4}{|c|}{ Propensity score matched } \\
\hline $\mathrm{RN}(N=2646)$ & 0.48 & 0.15 & 0.14 \\
\hline $\mathrm{NP}(N=1533)$ & 0.43 & 0.11 & 0.11 \\
\hline IRR & 0.90 & 0.73 & 0.79 \\
\hline$p$ value & 0.007 & 0.013 & 0.005 \\
\hline \multicolumn{4}{|c|}{ Propensity score matched and adjusted } \\
\hline $\begin{array}{l}\text { Incident rate ratio } \\
\text { RR) }\end{array}$ & 0.89 & 0.80 & 0.79 \\
\hline$p$ value & 0.016 & 0.004 & $<0.001$ \\
\hline
\end{tabular}

Restricting the study sample to the common support of the distribution of propensity scores reduced the sample of NP callers by 21 to 11533. Although a 2:1 match to RN callers was attempted, only $2646 R N$ callers met the common support and other restrictions. Propensity score model results do not include hospitalization due to small sample size and small number of hospitalizations

Propensity score was estimated using a logistic regression in which the dependent variable was an indicator for NP or RN routed call, with an estimated anti-parsimonious model. Independent variables included patient socio-demographics, comorbidity, and hospital, outpatient, and ED use in the 12 months prior to the call; the duration of the complaint; the urgency of the condition as defined by the triage software; patientreported pain score; and the number of complicating factors the patients identified. We also included interaction terms between the urgency of the condition and other independent variables. Each NP call was matched to $2 R N$ routed calls for the same chief complaint based on the propensity score derived from this model. Because urgency remained unbalanced in the propensity score-matched sample, we also estimated logistic and Poisson regression models that controlled for indicators of urgency, but the results remained unchanged

specialty care and ED visit. Compared with RN-triaged calls, calls triaged by NPs also had statistically significantly lower mean number of visits over 7 days for primary care, specialty care, and ED visits. No differences in healthcare use were observed past 7 days.

\section{DISCUSSION}

In this observational retrospective comparative effectiveness study, a program to integrate NPs into call center remote triage resulted in decreased 7-day healthcare use. The finding was consistent across adjusted analyses with propensity matching with reduced primary care, specialty care, and ED visits; hospitalizations were only analyzed in the adjusted counts with fewer events for NP routed calls. Reduction in healthcare use occurred on the same day and the day following the call with no differences observed in the following days. This reduction resulted in 0.7 primary care, 2.6 specialty care, 5.9 ED visits, and 1.4 hospitalizations avoided per 100 calls with similar chief complaints handled by NPs compared with RNs.
The impact of the addition of an NP in telephone triage systems has the potential to innovate and improve access to care. As call center procedures and telephone triage software are modified to incorporate NPs, and possibly physicians, there are potential benefits to patients, providers, and healthcare systems. Patients benefit by saving travel time and costly ED and clinic visits. Providers benefit by having patient concerns triaged in a way that either resolves the issue or directs the patient to the most appropriate level of care, obviating unnecessary in-person visits. Healthcare systems benefit by improving efficiency through a more informed and sophisticated telephone triage system. Such systems can train dedicated licensed independent providers, or use existing primary care and ED providers, to staff such a service and incorporate the role into their usual duties.

The greatest reduction in healthcare use was seen on the day of the call or the next day, suggesting a possible causal pathway. It is possible the NP provided reassurance that the severity of the problem did not require urgent care, allowing for routine follow-up in the appropriate clinical location beyond 7 days. The top three chief complaints (i.e., pain, respiratory, and urinary) accounted for over $80 \%$ of all calls handled by the NP. Because NPs had full prescribing authority, treatment such as antibiotics for suspected urinary tract infections or pain medications could be ordered to avoid an inperson visit. It is important to note that this pilot intervention did not result in increases in healthcare use, as some studies have shown. ${ }^{4}$ Considering the greater cost of NPs over RNs, it would be difficult to justify their use if there was no observable improvement in care.

The findings of this study add to the existing literature on remote telephone triage. Despite both patient's demand for telephone access to their healthcare providers and the system's desire to provide timely and efficient care, the existing literature has been mixed on the benefits of these systems. With some studies showing reductions in subsequent healthcare $\mathrm{use}^{3}$ and others showing either no improvement or greater use, ${ }^{4-9}$ the findings from this study should be contrasted with those. First, many of the prior studies have been from Europe, especially the UK, where differences in expectations of patients and providers may have impacted the results. Second, the design of this pilot project considered prior successful programs and lessons learned from the literature to inform the way calls were routed and handled by the NPs, potentially resulting in greater efficacy of the intervention. Lastly, there may be structural differences in the VHA healthcare system that resulted in this intervention being more effective.

The study findings have implications for healthcare policy. Integration of NPs into call centers should be considered in integrated healthcare systems to both improve access and also direct patients to the appropriate level of care. An expanded role in fee-for-service healthcare would require comprehensive reform of reimbursement rules for telemedicine in the USA, but which were recently modified during the COVID-19 pandemic. ${ }^{16}$ The importance of this triage role has been 


\section{Emergency Department}

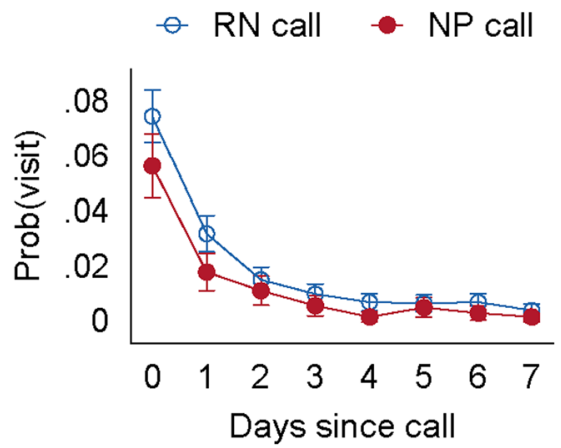

Specialty Care

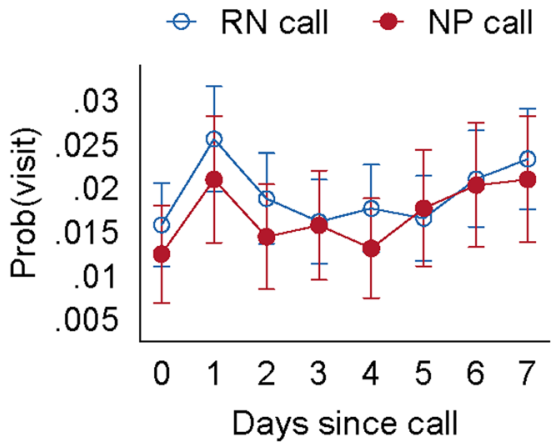

\section{Primary Care}

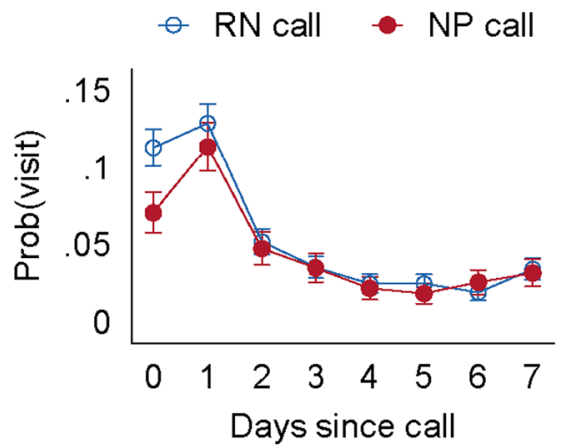

Figure 3 Propensity score-matched probability of healthcare use by day, type of use, and whether call was routed by an NP or RN. Propensity score model results do not include hospitalization due to small sample size and inability to adequately match.

heightened during the COVID-19 pandemic with reductions in in-person visits by half, but only a portion of those visits being replaced by telemedicine visits in both $\mathrm{VA}^{17}$ and private sector. ${ }^{18}$ Enhanced telephone triage can continue to fill that void. Cost avoidance to the healthcare system can be estimated based upon differences in costs of care between telehealth and in-person visits whether to a clinic, ED, or urgent care. Further adjustments to the TEDP triage system will allow NPs to handle a greater percentage and higher acuity of calls.

There are several limitations worth mentioning. First, the study was performed in an integrated healthcare system serving Veterans with limited third-party billing, an integrated EHR, and ability to provide care across state lines. Thus, the findings may not be generalizable. Second, patients were not randomly assigned to NPs or RNs, potentially introducing bias. This is intentional as the RNs selected less complex calls that they believe can be handled by the NPs to provide the needed care by phone, or triage to telephone only follow-up. Propensity score matching was used to overcome this bias, but some residual bias may remain. Because of this non-random allocation, patients handled by RNs had higher mean comorbidity scores and healthcare use. To overcome this bias, propensity score matching was used to select 2646 of the entire set of $48,024 \mathrm{RN}$ calls to match to the $1554 \mathrm{NP}$ calls. Third, all healthcare use outside of VHA was not captured, so it was possible there was differential non-VHA use between the two groups in the 7 days after the calls. Finally, there was no information available on patient satisfaction or preferences for phone care compared to in-person care.

\section{CONCLUSION}

This observational study demonstrated reduced 7-day healthcare use after a remote triage call to an NP compared to routine $\mathrm{RN}$ triage. This has important implications for the expansion of such systems and requires additional study to better explain the findings, identify which patients have the greatest potential for benefit, and understand patient preferences for such services.

Acknowledgments: This material is based upon work supported (or supported in part) by the Department of Veterans Affairs, Veterans Health Administration, Office of Nursing Services, VA Office of Rural Health, and the Office of Research and Development, Health Services Research and Development (HSR\&D) Service through the Comprehensive Access and Delivery Research and Evaluation (CADRE) Center (CIN 13-412). It was also undertaken as part of the Department of Veterans Affairs' Primary Care Analytics Team, supporting and evaluating VA's transition to a patient-centered medical home. Funding for the Primary Care Analytics Team is provided by the VA Office of Primary Care. Thank you to Emily Ashmore for the preparation of figures and Kelby Rewerts for editing assistance. 
Corresponding Author: Peter J. Kaboli, MD, MS; Veterans Rural Health Resource Center-Iowa City, VA Office of Rural Health, and Center for Access and Delivery Research and Evaluation (CADRE) at the Iowa City VA Healthcare System, Iowa City, IA, USA (e-mail: peter. kaboli@va.gov).

\section{Compliance with Ethical Standards:}

Conflict of Interest: The authors declare that they do not have a conflict of interest.

Disclaimer: The views expressed in this article are those of the authors and do not necessarily represent the views of the Department of Veterans Affairs or the United States Government.

Data: Available to researchers with VA accreditation.

Statistical Code: Available to interested readers by contacting the lead author.

Protocol: Available to interested readers by contacting the lead author.

\section{REFERENCES}

1. Boggan JC, Shoup JP, Whited JD, et al. Effectiveness of acute care remote triage systems: a systematic review. J Gen Intern Med. 2020.

2. Rushton S, Boggan J, Lewinski A, et al. Effectiveness of remote triage: systematic review. In: Evidence Synthesis Program HSRaDS, Office of Research and Development, Department of Veterans Affairs., ed. Vol VA ESP Project \#09-010. Washington, DC2019.

3. Munro J, Nicholl J, O'Cathain A, Knowles E. Impact of NHS direct on demand for immediate care: observational study. BMJ. 2000;321(7254):150153.

4. Campbell JL, Fletcher E, Britten N, et al. Telephone triage for management of same-day consultation requests in general practice (the ESTEEM trial): a cluster-randomised controlled trial and costconsequence analysis. Lancet. 2014;384(9957):1859-1868.

5. McKinstry B, Walker J, Campbell C, Heaney D, Wyke S. Telephone consultations to manage requests for same-day appointments: a randomised controlled trial in two practices. Br J Gen Pract. 2002;52(477):306-310.
6. Cragg DK, McKinley RK, Roland MO, et al. Comparison of out of hours care provided by patients' own general practitioners and commercial deputising services: a randomised controlled trial. I: The process of care. BMJ. 1997;314(7075): 187-189.

7. Richards DA, Godfrey L, Tawfik J, et al. NHS Direct versus general practice based triage for same day appointments in primary care: cluster randomised controlled trial. BMJ. 2004;329(7469):774.

8. Richards DA, Meakins $\mathbf{J}$, Tawfik $\mathbf{J}$, et al. Nurse telephone triage for same day appointments in general practice: multiple interrupted time series trial of effect on workload and costs. BMJ. 2002;325(7374):1214.

9. Turner J, O'Cathain A, Knowles E, Nicholl J. Impact of the urgent care telephone service NHS 111 pilot sites: a controlled before and after study. BMJ Open. 2013;3(11):e003451.

10. Elshove-Bolk J, Mencl F, van Rijswijck BT, Simons MP, van Vugt AB. Validation of the Emergency Severity Index (ESI) in self-referred patients in a European emergency department. Emerg Med J. 2007;24(3):170174.

11. DHSISystems. https://www.dshisystems.com/. Published 2020. Accessed May 13, 2020.

12. Gagne JJ, Glynn RJ, Avorn J, Levin R, Schneeweiss S. A combined comorbidity score predicted mortality in elderly patients better than existing scores. J Clin Epidemiol. 2011;64(7):749-759.

13. Dowd BE, Greene WH, Norton EC. Computation of standard errors. Health Serv Res. 2014;49(2):731-750.

14. Austin PC. Optimal caliper widths for propensity-score matching when estimating differences in means and differences in proportions in observational studies. Pharm Stat. 2011;10(2):150-161.

15. Stata Statistical Software: Release 15 [computer program]. College Station, TX: StataCorp LLC.; 2017.

16. Verma S. Early Impact of CMS Expansion of Medicare Telehealth During COVID-19. In. Vol 2020. Health Affairs Blog2020.

17. Baum A, Kaboli PJ, Schwartz MD. Reduced in-person and increased telehealth outpatient visits during the COVID-19 pandemic. Ann Intern Med. 2020

18. Patel SY, Mehrotra A, Huskamp HA, Uscher-Pines L, Ganguli I, Barnett ML. Trends in outpatient care delivery and telemedicine during the COVID-19 pandemic in the US. JAMA Intern Med. 2020.

Publisher's Note: Springer Nature remains neutral with regard to jurisdictional claims in published maps and institutional affiliations. 